\title{
Human vascular endothelial cells reduce sphingosylphosphorylcholine-induced smooth muscle cell contraction in co-culture system through integrin $\beta 4$ and Fyn
}

\author{
Di GE ${ }^{1}$, Ning MENG ${ }^{1}$, Le SU ${ }^{1,2}$, Yun ZHANG ${ }^{2}$, Shang-li ZHANG ${ }^{1,2}$, Jun-ying MIAO ${ }^{1,2}$, Jing ZHAO ${ }^{1,2, *}$ \\ ${ }^{1}$ Institute of Developmental Biology, School of Life Science, Shandong University, Ji-nan 250100, China; ${ }^{2}$ Key Laboratory of Cardiovas- \\ cular Remodeling and Function Research, Chinese Ministry of Education and Chinese Ministry of Health, Shandong University Qilu \\ Hospital, Ji-nan 250100, China
}

\begin{abstract}
Aim: In vascular strips, the adjacent endothelial cells modulate the contraction of vascular smooth muscle cells (VSMCs) induced by sphingosylphosphorylcholine (SPC) through nitric oxide (NO). The aim of this study was to elucidate the mechanisms by which vascular endothelial cells (VECs) reduce the SPC-induced contraction of VSMCs in a co-culture system.

Methods: Human umbilical VECs and VSMCs were co-cultured. The VECs were transfected with integrin $\beta 4-$ or Fyn-specific siRNA. The areas of VSMCs that are involved in cell contractility were quantified using the Leica confocal software and collagen contractility assay. The production of NO in VECs was measured in the cell supernatants using NO Detection Kit. The levels of integrin $\beta 4$ and Fyn in VECs and the levels of Rho kinase (ROCK) in VSMC were detected using immunofluorescence assays or Western blots.

Results: Co-culture with VECs reduced the contraction of VSMCs induced by SPC (30 $\mu \mathrm{mol} / \mathrm{L})$. The down-regulation of integrin $\beta 4$ or Fyn in VECs by the specific siRNA (20 nmol/L) was able to counteract the effects of VECs on the SPC-induced VSMC contractions. Furthermore, the integrin $\beta 4$-specific siRNA ( 20 and $40 \mathrm{nmol} / \mathrm{L}$ ) significantly reduced the level of Fyn protein and the production of NO in VECs, while increased the level of ROCK in VSMCs that had been stimulated by SPC.

Conclusion: The VECs reduced the SPC-induced contraction of VSMCs in the co-culture system through integrin $\beta 4$ and Fyn proteins. In this process, NO may be the factor downstream of integrin $\beta 4$ in VECs, while ROCK may be the key protein regulating the contraction of VSMCs.
\end{abstract}

Keywords: human umbilical vein; vascular endothelial cells; vascular smooth muscle cells; integrin $\beta 4$; Fyn; contraction; sphingosylphosphorylcholine

Acta Pharmacologica Sinica (2012) 33: 57-65; doi: 10.1038/aps.2011.142; published online 5 Dec 2011

\section{Introduction}

Vasoconstriction that is caused by the tonic contraction of vascular smooth muscle cells in large arteries may invoke vasospasms, which are not only peculiar occurrences that are limited to arcane forms of angina, acute myocardial infarction and sudden cardiac death but also acute events that promote thrombosis, atherosclerotic plaque ruptures and mural plaque hemorrhages ${ }^{[1-4]}$. It has been reported that the $\mathrm{Ca}^{2+}$ sensitization of contractile proteins is the principal event that induces vasospasms ${ }^{[5]}$. Therefore, research on the $\mathrm{Ca}^{2+}$ sensitizationinduced contraction of vascular smooth muscle cells (VSMCs)

\footnotetext{
* To whom correspondence should be addressed.

E-mail jingzhao@sdu.edu.cn

Received 2011-02-28 Accepted 2011-09-19
}

could greatly contribute to understanding the mechanism underlying vasospasms.

The lysosphingolipid sphingosylphosphorylcholine (SPC) is an important cardiovascular mediator that is derived from sphingomyelin. It mainly associates with high-density lipoproteins (HDLs) in plasma and has atheroprotective effects via its actions on endothelial cells in vivo. However, when endothelial cells are damaged, SPC may target VSMCs and contribute to cardiovascular disease ${ }^{[6]}$. Recently, high concentrations of SPC $(30 \mu \mathrm{mol} / \mathrm{L})$ have been reported to be novel messengers for the Rho kinase-mediated $\mathrm{Ca}^{2+}$ sensitization of VSMC contraction in vitro, and thus SPC has been used as a tool to research $\mathrm{Ca}^{2+}$ sensitization-induced vasospasms ${ }^{[7]}$. It is now clear that SPC may increase nitric oxide production in VECs and subsequently reduce the contraction of smooth 
muscle cells in the intact vascular strips ${ }^{[8]}$, which may be the main mechanism for the cardiovascular protection that is provided by SPC via its actions on endothelial cells in vivo. However, the identities of the upstream mediators of the known pathway that are involved in the reduction of SPC-induced vasospasms by endothelial cells have only been speculated.

Integrin $\beta 4$, which possesses an exceptionally large cytoplasmic domain, plays a crucial role in hemidesmosome assembly by binding to extracellular matrix (ECM) proteins in the basement membrane zones of epithelial tissues ${ }^{[9]}$. Our recent research has shown that this membrane protein plays an important role in the regulation of vascular endothelial cell functions, such as apoptosis, differentiation and senescence ${ }^{[10-12]}$. Furthermore, in our previous research, integrin $\beta 4$ was associated with NO production ${ }^{[12]}$. Therefore, in this study, we attempted to elucidate whether and how the integrin $\beta 4$ in VECs is involved in the VEC-modulated VSMC contraction induced by SPC.

Fyn is a member of the Src family of protein tyrosine kinases that is widely expressed in various types of cells and has greater functional redundancy compared to most of the other SrcPTK members ${ }^{[13,14]}$. Recent reports have indicated that the co-culturing of endothelial and smooth muscle cells affects the gene expression of angiogenic factors in VECs ${ }^{[15]}$, suggesting the important roles of angiogenic factors in endothelial/ smooth muscle interactions. In addition, Fyn is an important factor in endothelial cell capillary morphogenesis ${ }^{[16]}$. However, the possible involvement of the VEC-derived Fyn in the contraction of VSMCs is not yet clear.

Here, we report that VECs reduce the SPC-induced contraction of VSMCs in the co-culture system of VECs and VSMCs. The VECs perform this function through the integrin $\beta 4$ and Fyn proteins. Moreover, NO and Fyn may be the factors downstream of integrin $\beta 4$ in the reduction of SPC-induced VSMC contraction that is caused by the VECs, and ROCK in the VSMCs may be the key protein that regulates VSMC contraction.

\section{Materials and methods Materials}

M199 medium and fetal bovine serum (FBS) were purchased from Hyclone Lab Inc, USA. Fibroblast growth factor (FGF) was purchased from EssexBio Group, China. SPC was obtained from Sigma Co, USA and was dissolved in ethanol, and a concentration of $30 \mu \mathrm{mol} / \mathrm{L}$ was applied to the cells. This concentration was previously used for the promotion of the contraction of arteries and rat VSMCs in vitro ${ }^{[7,17,18]}$. The primary antibodies (mouse anti-human integrin $\beta 4$ or Fyn and rabbit anti-human ROCK), secondary antibodies (goat antimouse TR antibody and goat anti-rabbit FITC antibody) and the specific small interfering RNA (siRNA) oligonucleotides of human integrin $\beta 4$ (SC-35678) were all purchased from the Santa Cruz Co, USA. The human Fyn siRNA was synthesized by Qiagen, USA. The following primers were used: human Fyn sense RNAi, 5'-GAGUAUAUGAACAAAGGAA-3' and human Fyn antisense RNAi, 5'-UUCCUUUGUUCAUAUA-
CUC-3'. The RNAiFect ${ }^{\mathrm{TM}}$ Transfection Reagent, $\beta$-actin siRNA and rhodamine-labeled siRNA were all purchased from Qiagen, USA. All other reagents were ultrapure grade products from China.

\section{Culturing of VSMCs}

This investigation conformed to the Declaration of Helsinki. The VSMCs were cultured as previously described ${ }^{[19]}$. In brief, human umbilical veins were excised; the endothelium was stripped away; the media of the veins were cut into small pieces and placed into $35 \mathrm{~mm}$ culture plates that had been coated with lumens; and these pieces were grown in M199 medium with $10 \%$ fetal calf serum and $2 \mathrm{ng} / \mathrm{mL} \mathrm{FGF}-2$ at $37^{\circ} \mathrm{C}$ in $5 \% \mathrm{CO}_{2}$ and $95 \%$ air. Every $72 \mathrm{~h}$ thereafter, the medium was replaced with fresh, complete M199. Cells appeared from explants within 2 weeks and grew to confluence in approximately 4 weeks. The identities of the cultured cells as smooth muscle cells were confirmed by the "hill and valley" patterns of cell growth and by positive staining for smooth muscle a-actin (a-SMA). The experiments were performed on cells that had undergone 5-8 passages.

\section{Culturing of HUVECs}

Human umbilical vein endothelial cells (HUVECs) were obtained as previously described ${ }^{[20]}$. Cells were grown in M199 medium with $10 \%$ fetal calf serum and $2 \mathrm{ng} / \mathrm{mL}$ of FGF-2. The experiments were performed on cells that had undergone 10-20 passages.

\section{Co-culturing of VECs and VSMCs}

In the direct co-culture system, VECs were grown to confluence on the bottom of a 24-well plate, and then the VSMC suspension was adjusted to $1.7 \times 10^{4}$ cells $/ \mathrm{mL}, 1 \mathrm{~mL}$ of which was placed on the confluent monolayer of endothelial cells. After the VECs and VSMCs were co-cultured for $24 \mathrm{~h}$, the medium was replaced with basal M199 medium (without FBS or FGF-2). Then, the separate co-cultures were treated as follows: (a) cultured in M199 medium with FBS and FGF-2 (normal); (b) cultured in M199 medium with ethanol at 0.3\% (v/v) (control) and no serum or FGF-2; and (c) cultured in basal M199 medium with SPC $(30 \mu \mathrm{mol} / \mathrm{L})$. The contractile changes of the cells were quantified using the Leica software, which analyzed the images at $1 \mathrm{~h}$.

To observe the changes in VSMC morphology clearly, an additional micropore membrane co-culture system was also used $^{[21]}$. Briefly, the VECs and VSMCs were seeded onto the upside of microporous filtering membranes in 48-well plates at a density of $8.5 \times 10^{3}$ cells $/ \mathrm{mL}$. Each membrane consisted of a mixed cellulose ester with a $0.45 \mu \mathrm{m}$ micropore that was precoated with matrigel. After allowing $48 \mathrm{~h}$ for attachment, the membrane was placed with the VEC side down into a 48-well plate containing VSMCs. After the VECs and VSMCs were cocultured for $24 \mathrm{~h}$, the medium was replaced with basal M199 medium (without FBS or FGF-2). Then, the separate co-cultures were treated as follows: culturing in M199 medium with ethanol at $0.3 \%(v / v)$ (control) and culturing in basal M199 
medium with SPC $(30 \mu \mathrm{mol} / \mathrm{L})$. The cell morphologies were observed using a phase contrast microscope (Nikon, Japan).

\section{Immunofluorescence assay}

The VSMCs were co-cultured with VECs in the micropore membrane insert well system for $24 \mathrm{~h}$, and then an immunofluorescence assay was performed on the sub-confluent VECs and VSMCs to examine the expression of integrin $\beta 4$ and Fyn in the VECs and ROCK in the VSMCs as previously described ${ }^{[10]}$. After the addition of the primary antibodies (mouse anti-human integrin $\beta 4$, Fyn or a-SMA IgG and rabbit anti-human ROCK IgG) and appropriate secondary antibodies (goat anti-mouse-TR or goat anti-rabbit-FITC), the samples were evaluated by laser scanning confocal microscopy (LSCM) (Leica, Germany). We randomly selected the region of interest and then zoomed in on the same frames. The relative fluorescent intensity per cell was calculated as the total value of the sample in the zoom scan divided by the total number of cells (at least 200 cells) in the same scan. The mean areas of a-SMApositive cells (smooth muscle cells) that showed contractility were quantified (at least 200 cells). The percent reduction in cell area $(\%)=($ mean area of treated cells $/$ mean area of normal cells) $\times 100 \%$.

\section{RNA interference}

RNA interference (RNAi) was performed as described previously ${ }^{[12]}$. Briefly, cells that were at approximately $60 \%$ confluence were transfected with scramble RNA (negative control) or siRNA against integrin $\beta 4(10-40 \mathrm{nmol} / \mathrm{L})$ or Fyn $(60 \mathrm{nmol} / \mathrm{L})$ using the RNAiFect Transfection Reagent according to the manufacturer's instructions. After $24 \mathrm{~h}$, the medium was replaced with M199 medium supplemented with serum and FGF-2. Cells were cultured for another $24 \mathrm{~h}$ for the desired assays. Western blot analyses were conducted to estimate the effects of gene silencing.

\section{Western blotting}

The Western blots were performed as previously described ${ }^{[10]}$. Briefly, equal amounts of total proteins were loaded onto $7.5 \%$ SDS-polyacrylamide gels and electrophoretically transferred to nitrocellulose transfer membranes. After blocking with 5\% skim milk in PBS and 0.5\% (v/v) Tween 20 for $1 \mathrm{~h}$, the membranes were incubated with monoclonal Fyn or the integrin $\beta 4$ protein antibody (mouse anti-human) overnight at $4{ }^{\circ} \mathrm{C}$ and then incubated with HRP-linked secondary antibodies (goat anti-mouse) for $1 \mathrm{~h}$ at room temperature (RT), followed by color development with $0.06 \%$ DAB (diaminobenzidine) and $0.03 \% \mathrm{H}_{2} \mathrm{O}_{2}$ in PBS for 3-5 min at RT. Distilled water was used to stop the reaction. The monoclonal anti- $\beta$-actin antibody (mouse anti-human) was used to ascertain that equal protein amounts were loaded. The relative quantities of the proteins were analyzed using the ImageTool software.

\section{Cell contraction analysis}

Collagen gels were prepared from rat tail type 1 collagen and seeded in 24-well culture dishes. Each well contained $400 \mu \mathrm{L}$ collagen (final collagen concentration of $1.5 \mathrm{mg} / \mathrm{mL}$ ), $100 \mu \mathrm{L}$ neutralization solution and $1 \times 10^{5}$ VSMCs based on the technique that was described previously ${ }^{[22-24]}$. The VSMCs in the collagen gels were allowed to equilibrate overnight in M199 medium. The VECs grew to confluence on the bottom of the 24-well plates, and the collagen gel in which the VSMCs were embedded was placed on the confluent monolayer of endothelial cells. After the VECs and VSMCs were co-cultured for $24 \mathrm{~h}$, they were used for the SPC treatment. Gel images were captured at $2 \mathrm{~h}$ using a camera, and the areas $\left(\mathrm{cm}^{2}\right)$ of the gels were measured using the Image J software (http://rsb.info. nih.gov/ij/). The original area of a collagen gel was represented by the area $\left(2 \mathrm{~cm}^{2}\right)$ of a well from a 24-well plate. A decrease in the gel area correlates with an increase in contractility. Changes in contractility that resulted from the experimental treatments were expressed as the percent of the gel area that was observed relative to that of the untreated control. Three fields were analyzed for each experiment that was performed.

\section{NO level assay}

After the VECs were treated with the siRNA for $48 \mathrm{~h}$ and stimulated with SPC for 0, 3, 15, and $30 \mathrm{~min}$, the supernatant was used for a NO level assay using the NO Detection Kit (Nanjing Jiancheng, China) according to the manufacturer's instructions. The optical density was measured at $550 \mathrm{~nm}$ (wavelength). The level of $\mathrm{NO}$ was expressed as $\mu \mathrm{mol} / \mathrm{L}$.

\section{Cell viability assay}

HUVECs were plated in 96-well cell culture plates. Cells that were at approximately $60 \%$ confluence were transfected with scramble RNA (negative control) or integrin $\beta 4$ siRNA (40 $\mathrm{nmol} / \mathrm{L}$ ). After $24 \mathrm{~h}$, the medium was replaced with M199 medium for another $24 \mathrm{~h}$. Then, the cells were treated with basal M199 medium to which $0.3 \%$ ethanol $(v / v)$ or SPC was added for $1 \mathrm{~h}$, and the cell viability was assayed by the MTT assay as previously described ${ }^{[10]}$. The viability $(\%)=(O D$ of treated group/OD of control group) $\times 100 \%$.

\section{Statistic analysis}

Data were expressed as mean \pm SEM and accompanied by the number of experiments that were performed independently. A statistical analysis was conducted using a one-way ANOVA, and differences of $P<0.05$ were considered to be statistically significant.

\section{Results \\ VECs reduced SPC-induced VSMC contraction in co-culture systems \\ The VECs and VSMCs were co-cultured in the micropore membrane insert well co-culture system for $24 \mathrm{~h}$. Then, the VSMCs were treated with SPC for 30, 45, or $60 \mathrm{~min}$, and the cell contraction morphologies were observed under a micro- scope. The images show that the single-culture SPC treatment decreased the area of each individualVSMC significantly at 60 min compared with cocultured ones, but the same treatment}


failed to decrease the areas of the VSMCs in the co-cultures (Figure 1A).

After the VSMCs were treated with SPC for $1 \mathrm{~h}$, the cell contraction morphologies were observed using an immunofluorescence assay. The results showed that SPC caused the a-SMA-positive VSMC layer to shrink in the single-cultures, but this phenomenon was not observed in the co-cultures. As shown in the column (Figure 1B), the average length of the VSMCs in the co-cultured systems was significantly longer than that in the single-cultured ones $(P<0.01$ and $P<0.01)$. These results indicate that VECs inhibit SPC-induced VSMC contraction.

In addition, we examined the VSMCs using a collagen contractility assay, and the images show that the SPC treatment of the single-cultures decreased the collagen-containing areas, but the same treatment failed to decrease these areas in the co-cultures $(P<0.01$ and $P<0.01)$ (Figure $1 C)$. Thus, the VECs reduced VSMC contraction in co-cultures that had been stimulated with SPC.

\section{Integrin $\beta 4$-knockdown VECs failed to show reduced SPC-induced} VSMC contraction

To identify integrin $\beta 4$ in VECs that had been co-cultured with VSMCs, we measured integrin $\beta 4$ protein levels in single-cultured and co-cultured VECs. As shown in Figure 2A, the level of integrin $\beta 4$ was higher in the co-cultured VECs than that in the single-cultured ones $(P<0.05)$, indicating a possible role for integrin $\beta 4$ in the interaction between VECs and VSMCs.

To further elucidate the role of integrin $\beta 4$ in the effects of VEC s on SPC-induced VSMC contraction, RNAi was used. As shown in Figure 2B, integrin $\beta 4$ siRNA administered at 20 $\mathrm{nmol} / \mathrm{L}$ clearly downregulated integrin $\beta 4$ expression at $48 \mathrm{~h}$ $(P<0.01)$. After the VECs were transfected with integrin $\beta 4$ siRNA or scramble siRNA for $24 \mathrm{~h}$, the medium was changed, and the VECs were co-cultured with VSMCs as described above. As shown in Figure 2C and 2D, after the VSMCs were treated with SPC for $1 \mathrm{~h}$, the areas of the VSMCs and collagens that had been co-cultured with the integrin $\beta 4$-knockdown VECs were significantly smaller than those of the VSMCs that

\section{A} SPC

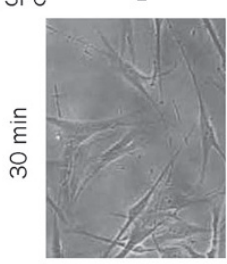

VSMC
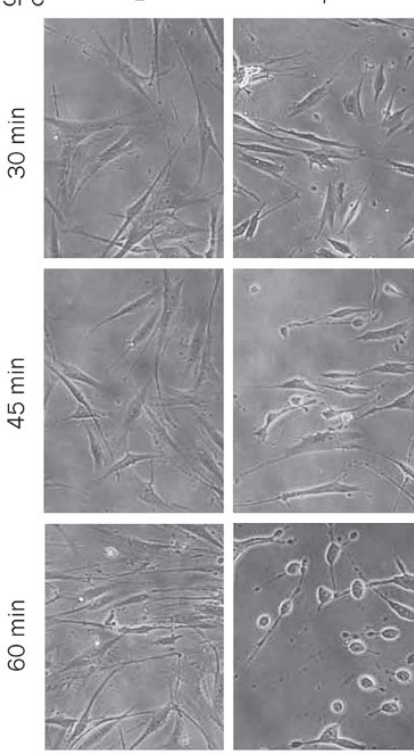

VSMC+VEC
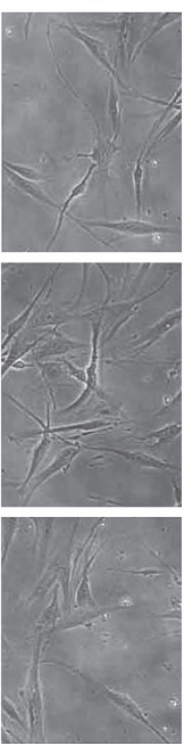

B

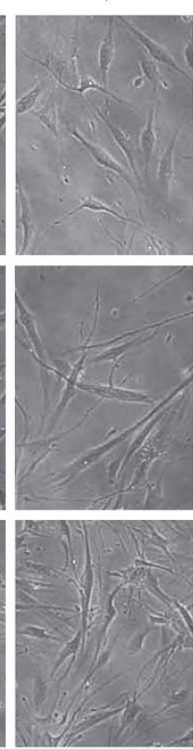

C
VSMC

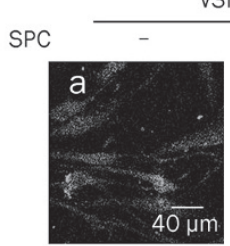

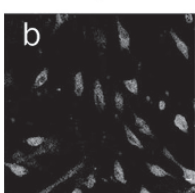
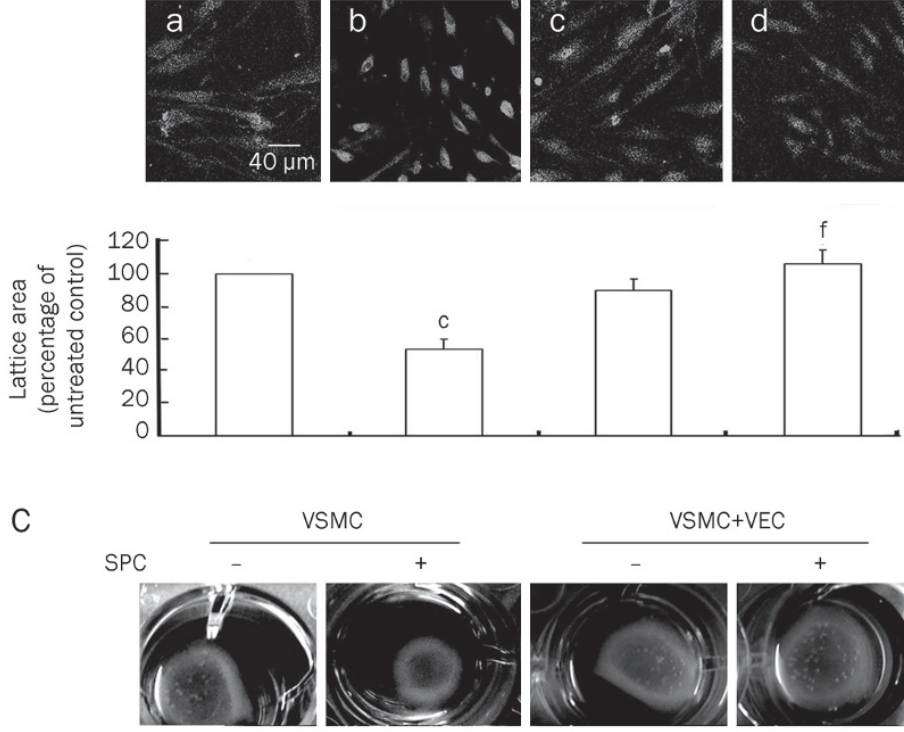

VSMC+VEC

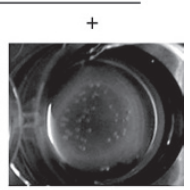

VSMC+VEC

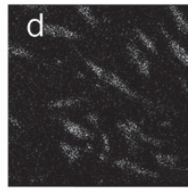

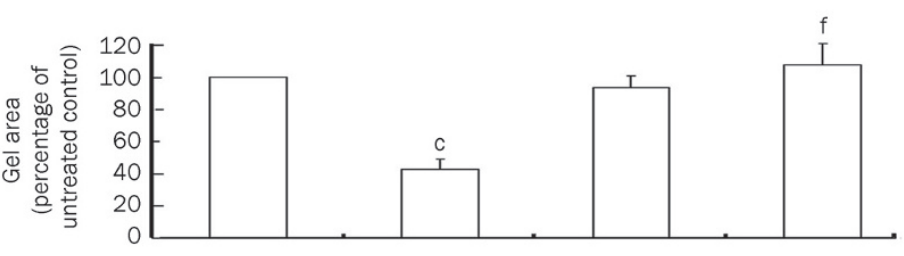

Figure 1. VECs inhibited the SPC-induced contraction of VSMCs. Cells were preincubated for 30,45 , or 60 min at $37^{\circ} \mathrm{C}$ in basal M199 medium with 30 $\mu \mathrm{mol} / \mathrm{L}$ SPC, and the morphology of the VSMCs in the micropore membrane insert well system was observed under a phase contrast microscope $(\times 200)$ (A). In the direct co-cultured systems that had been preincubated for 60 min with SPC, after staining with $\alpha$-SMA, the areas of $\alpha$-SMA-positive cells were also analyzed using the laser confocal software (B). The bar graph shows the relative contractilities following the SPC treatment, which are represented as the ratios of the average SMC area to that of the untreated control $\left({ }^{c} P<0.01\right.$ vs SPC-non-stimulated VSMCs; ${ }^{f} P<0.01$ vs SPC-stimulated VSMCs, $n=3$ ). (C) VSMCs in collagen gels were co-cultured with VECs and preincubated with SPC as mentioned above, and then the gel images were captured at $2 \mathrm{~h}$, and the areas $\left(\mathrm{cm}^{2}\right)$ of the gels were measured. The bar graph shows the relative contractility following SPC treatment, which is represented as the ratio of the gel area to that of the untreated control ( ${ }^{c} P<0.01$ vs SPC non-stimulated VSMCs; ${ }^{f} P<0.01$ vs SPC stimulated VSMCs, $\left.n=3\right)$. 
A
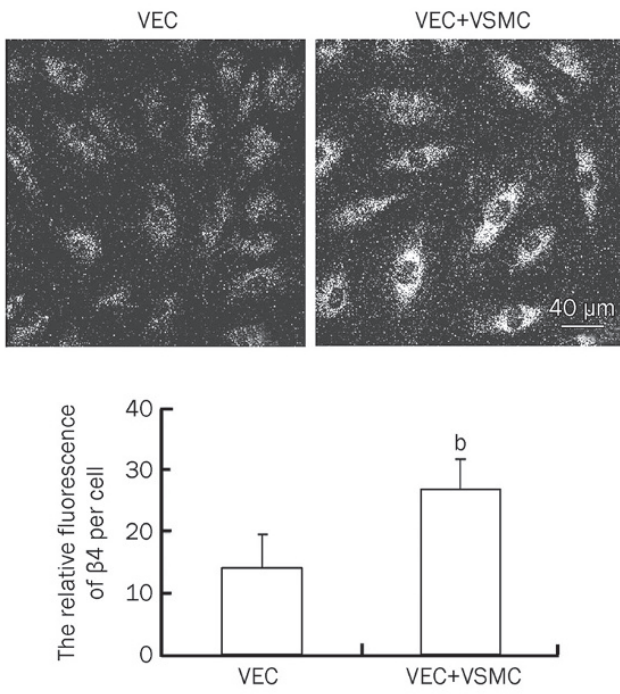

C
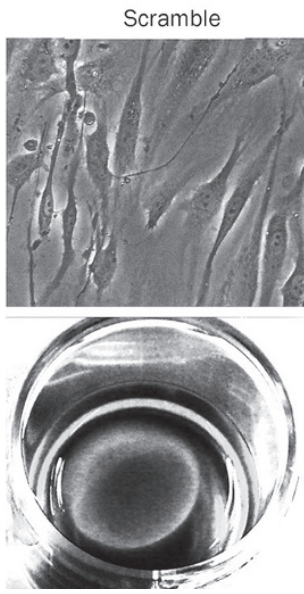

B4 SiRNA
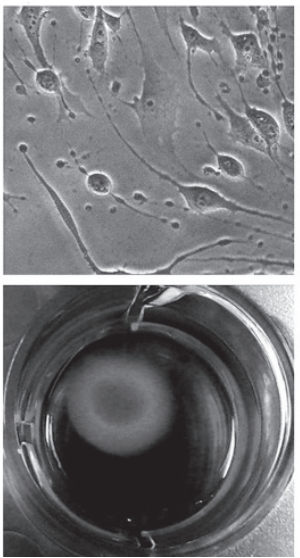

B
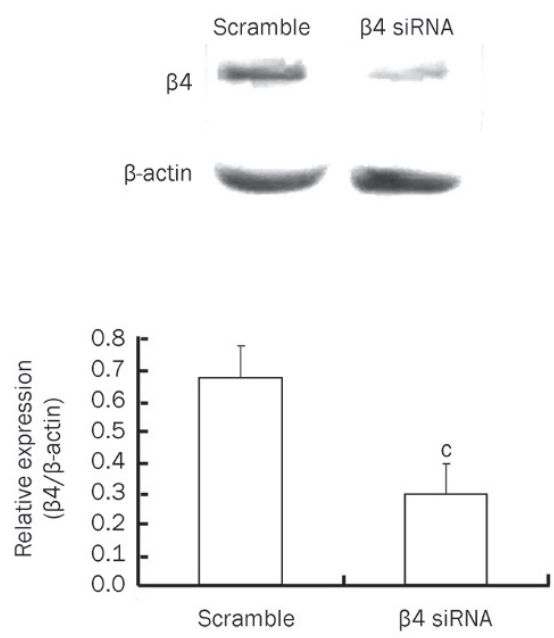

D

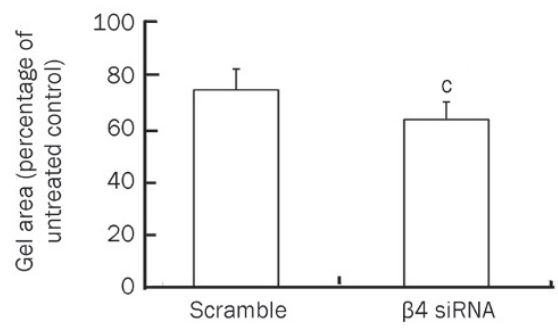

Figure 2. Effects of integrin $\beta 4$ knockdown in VECs on VSMC contraction. (A) VECs and VSMCs were co-cultured in the micropore membrane insert well co-culture system for $24 \mathrm{~h}$. Then, the levels of integrin $\beta 4$ in the VECs were examined using the immunofluorescence assay. The bar graph shows the relative intensity of integrin $\beta 4$ in the single-cultured and co-cultured VECs $\left({ }^{b} P<0.05\right.$ vs single-cultured VECs, $\left.n=3\right)$. (B) SiRNA-mediated down-regulation of integrin $\beta 4$ in VECs. The levels of integrin $\beta 4$ were determined by Western blotting $48 \mathrm{~h}$ after the start of the siRNA treatment. In the scramble control group (scramble ctrl), cells were transfected with scramble control siRNA ( ${ }^{\circ} P<0.01$ vs scramble ctrl, $n=3$ ). (C) VECs were treated with 20 nmol/L integrin $\beta 4$-specific siRNA or with scramble siRNA for $48 \mathrm{~h}$. After the addition of SPC, the effects of the integrin $\beta 4$ knockdown on the contraction of the VSMCs in the micropore membrane insert well system were observed under a phase contrast microscope $(\times 200)$ and analyzed by the collagen contractility assay. (D) The bar graph shows the relative contractility following SPC treatment, which is represented as the ratio of the gel area to that of the untreated control ( ${ }^{\mathrm{P}} P<0.01$ vs SPC-stimulated scramble ctrl, $\left.n=3\right)$.

had been co-cultured with the VECs that were transfected with the scramble siRNA $(P<0.01)$. Thus, the siRNA-mediated down-regulation of integrin $\beta 4$ in VECs could block the SPCinduced contraction of the VSMCs.

\section{Fyn-knockdown VECs failed to show reduced SPC-induced VSMC contraction}

To analyze the possible actions of Fyn in VECs that had been co-cultured with VSMCs, we measured the levels of this protein in single-cultured and co-cultured VECs. As shown in Figure 3A, the Fyn levels were higher in the co-cultured VECs than in the single-cultured VECs $(P<0.05)$, which is indicative of a possible key role of Fyn in the interaction between VECs and VSMCs.

As shown in Figure 3B, the expression levels of Fyn in the VECs that were transfected with Fyn siRNA at $60 \mathrm{nmol} / \mathrm{L}$ for $48 \mathrm{~h}$ were down-regulated compared with those in the untransfected VECs $(P<0.01)$. After the VECs were transfected with Fyn siRNA for $24 \mathrm{~h}$, the medium was changed, and the VECs were co-cultured with VSMCs as described above. As shown in Figure 3C and 3D, after the VSMCs were treated with SPC for $1 \mathrm{~h}$, the areas of the VSMCs and collagens that had been co-cultured with the Fyn-knockdown VECs were significantly smaller compared than those of the VSMCs that had been co-cultured with the scramble-siRNA-transfected VECs $(P<0.01)$. These results show that the siRNA-mediated down- 
A
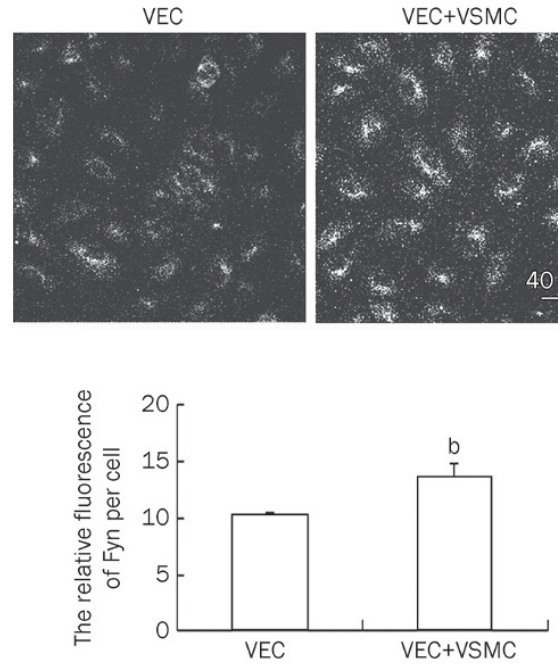

C
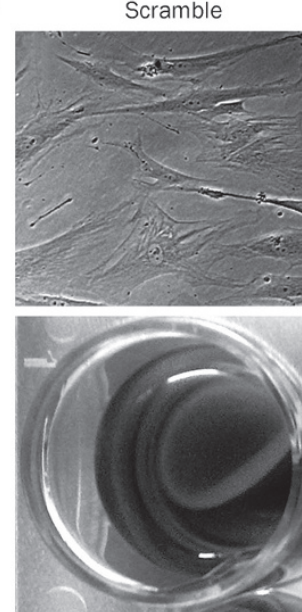

VEC+VSMC

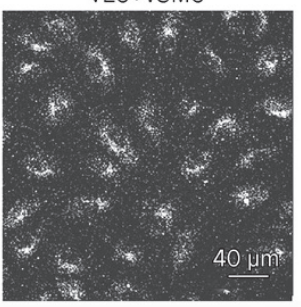

Fyn SiRNA
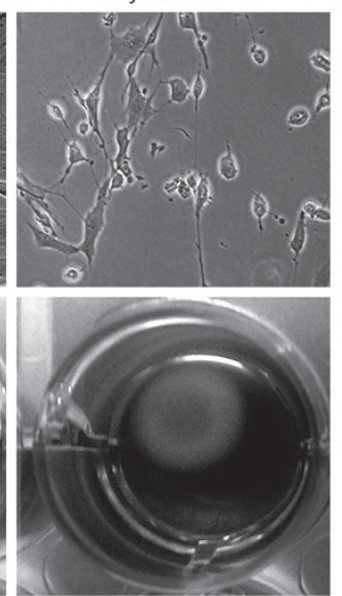

B
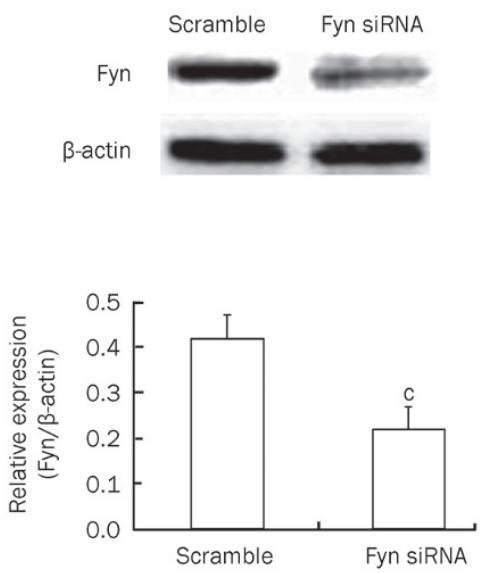

D

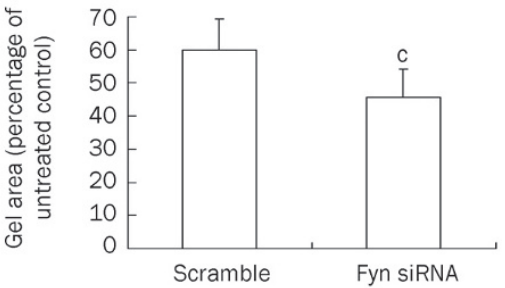

Figure 3. Effects of Fyn knockdown in VECs on VSMC contraction. (A) VECs and VSMCs were co-cultured in the micropore membrane insert well coculture system for $24 \mathrm{~h}$. Then, the levels of Fyn in the VECs were examined using the immunofluorescence assay. The bar shows the relative intensity of Fyn in single-cultured and co-cultured VECs $\left({ }^{b} P<0.05\right.$ vs single-cultured VECs, $\left.n=3\right)$. (B) SiRNA-mediated down-regulation of Fyn in VECs. The value of Fyn was determined by Western blotting $48 \mathrm{~h}$ after the start of the siRNA treatment. In the scramble control group (scramble ctrl), the cells were transfected with scramble control siRNA $\left({ }^{\circ} P<0.01\right.$ vs scramble ctrl, $n=3$ ). (C) VECs were treated with 20 nmol/L Fyn-specific siRNA or with scramble siRNA for $48 \mathrm{~h}$. After the SPC was added, the effects of the Fyn knockdown on the contraction of the VSMCs in the micropore membrane insert well system were observed under a phase contrast microscope $(\times 200)$ and analyzed using the collagen contractility assay. (D) The bar graph shows the relative contractility following SPC treatment, which is represented as a ratio of the gel area to that of the untreated control $\left({ }^{\circ} P<0.01\right.$ vs SPC-stimulated scramble ctrl, $n=3$ ).

regulation of Fyn in VECs could also counteract the inhibitory effects of the VECs on SPC-induced VSMC contraction.

\section{Knockdown of integrin $\beta 4$ inhibited Fyn expression in VECs}

An integrin $\beta 4$ knockdown strategy was used to elucidate the possible relationship between integrin $\beta 4$ and Fyn in the VECs. The results show that the knockdown of integrin $\beta 4$ inhibits the expression of Fyn $(P<0.05$ and $P<0.01)$ (Figure 4).

\section{Knockdown of integrin $\beta 4$ inhibited NO production in VECs}

To understand the underlying mechanism by which integrin $\beta 4$ participates in the effects of VECs on SPC-induced VSMC contraction, we analyzed the relationship between integrin $\beta 4$ and NO, the latter of which was the key factor in the VECreduced VSMC contraction stimulated by SPC. The results show that SPC promotes the production of $\mathrm{NO}$ at 3 and $15 \mathrm{~min}$ in VECs, whereas the knockdown of integrin $\beta 4$ inhibits SPCinduced NO production $(P<0.05)$ (Figure $5 \mathrm{~A})$.

\section{Knockdown of integrin $\beta 4$ did not change viability of VECs}

To clarify whether the knockdown of integrin $\beta 4$ abolishes VEC function as related to SPC-induced VSMC contraction by decreasing cell numbers, we assessed the viabilities of the VECs before and after the integrin $\beta 4$ siRNA treatment. 

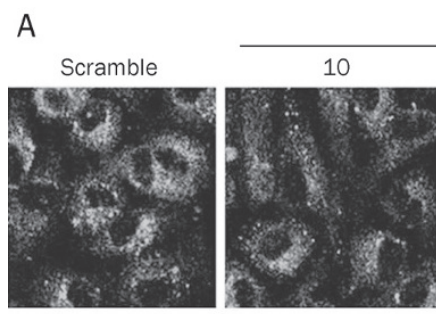

B4 siRNA (nmol/L)
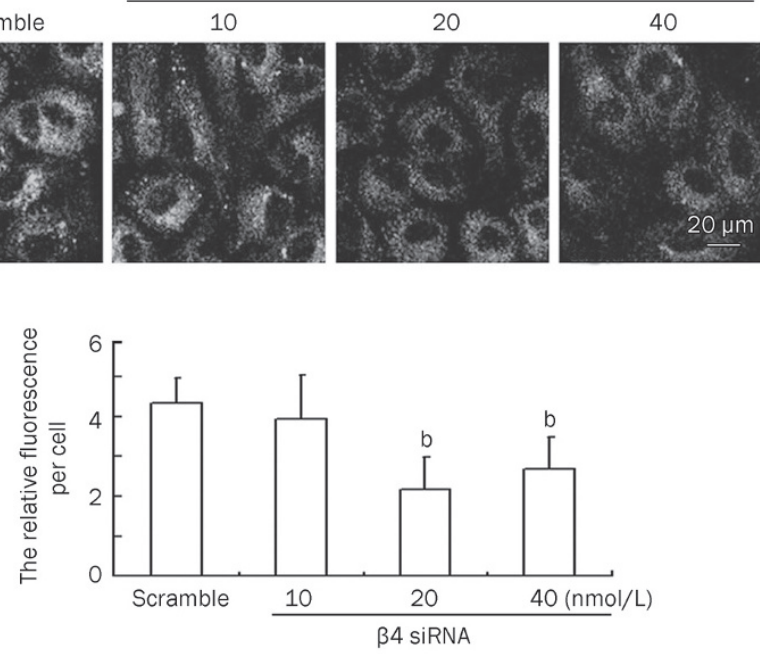

B
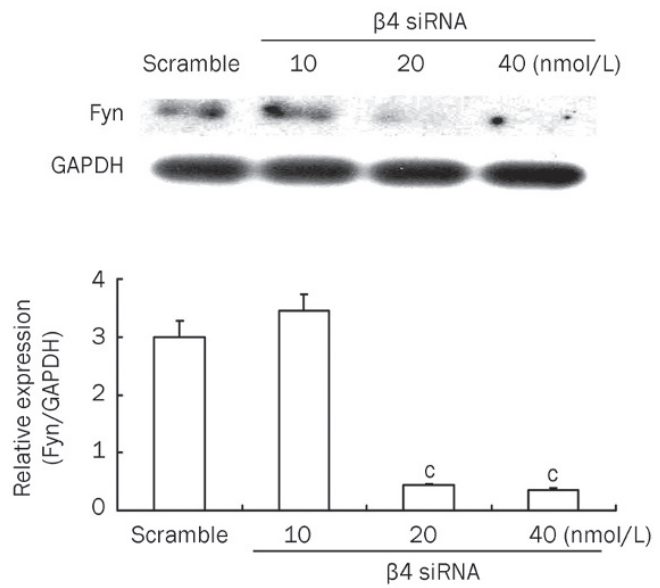

Figure 4. Effects of integrin $\beta 4$ knockdown on the changes in Fyn levels in VECs. (A) VECs were treated with $10-40 \mathrm{nmol} / \mathrm{L}$ integrin $\beta 4$ siRNA or scramble siRNA for $48 \mathrm{~h}$. The levels of integrin $\beta 4$ in the VECs were determined by the immunofluorescence assay, and the bar graph shows the relative fluorescent intensity of integrin $\beta 4$ per cell as determined by laser scanning confocal microscopy $\left({ }^{b} P<0.05\right.$ vs scramble ctrl, $\left.n=3\right)$. (B) After $10-40 \mathrm{nmol} / \mathrm{L}$ integrin $\beta 4$ siRNA or scramble siRNA were treated for $48 \mathrm{~h}$, the Fyn levels in the VECs were assessed using Western blotting $\left({ }^{c} P<0.01\right.$ vs scramble ctrl, $\left.n=3\right)$.

The results show that the knockdown of integrin $\beta 4$ does not change the viabilities of the VECs (Figure 5B).

ROCK levels in VSMCs that were treated with SPC and cocultured with integrin $\beta 4$-knockdown VECs were increased by SPC

Because the translocation of Rho kinase (ROCK) has been shown to participate in SPC-induced VSMC contraction ${ }^{[18]}$, we further analyzed the changes in ROCK levels in relation to VSMC contraction. As shown in Figure 6, when VSMCs were treated with SPC and when SPC-treated VSMCs were co-cultured with integrin $\beta 4$-knockdown VECs, contraction occurred, and the levels of ROCK in the VSMCs significantly
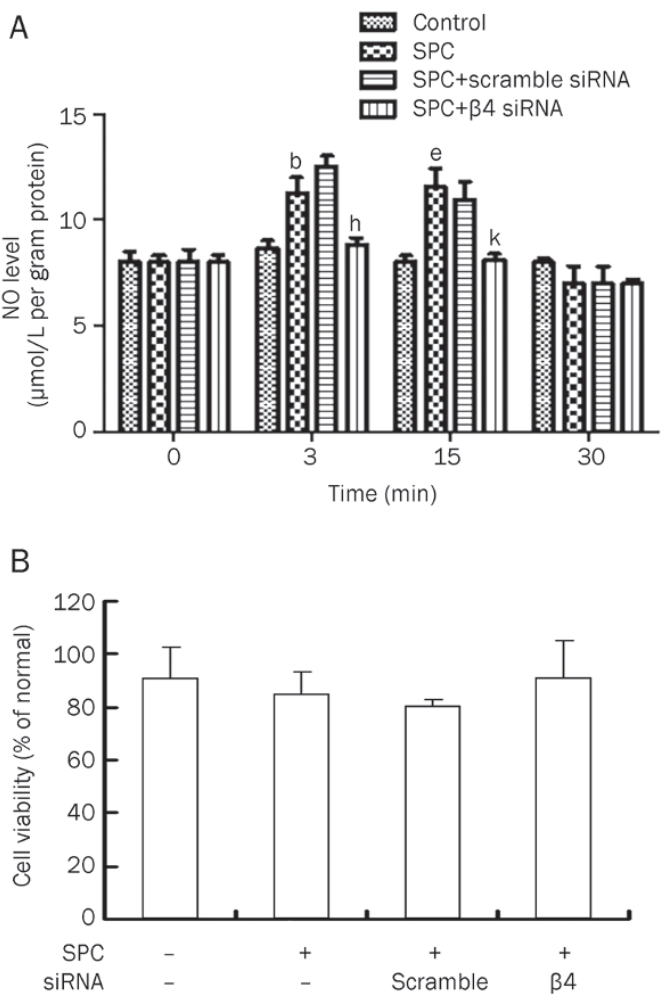

Figure 5. Effects of integrin $\beta 4$ knockdown on NO production in VECs. (A) VECs were cultured under normal conditions or with siRNA for $48 \mathrm{~h}$ and stimulated with SPC for $0,3,15$, or $30 \mathrm{~min}$, and the supernatant was used for the NO level assay using the NO Detection Kit. In the control group (ctrl), the cells were cultured in M199 medium with $0.3 \%(\mathrm{~V} / \mathrm{V})$ ethanol instead of SPC. In the scramble control group (scramble ctrl), cells were transfected with scramble control siRNA in the presence of SPC. The bar graph shows the changes in NO levels in VECs that had been treated with SPC $\left({ }^{b} P<0.05\right.$ vs ctrl at $3 \mathrm{~min},{ }^{e} P<0.05$ vs ctrl at $15 \mathrm{~min}$, $n=5)$ or with siRNA in the presence of SPC $\left({ }^{\mathrm{h}} P<0.05\right.$ vs scramble ctrl at 3 min, ${ }^{k} P<0.05$ vs scramble ctrl at $\left.15 \mathrm{~min}, n=5\right)$. (B) The viabilities of VECs were measured by MTT, and no significant changes were observed $(n=3)$.

increased compared to the corresponding controls $(P<0.05)$.

\section{Discussion}

Recently, SPC has been regarded as a novel messenger for the Rho kinase-mediated $\mathrm{Ca}^{2+}$ sensitization of VSMC contraction $^{[7]}$, but its effects on VSMCs depend on the presence of VECs. In this study, to obtain further information regarding the mechanism by which VECs inhibit SPC-induced VSMC contraction, we utilized a co-culture system containing VECs and VSMCs. The results show that VECs inhibit SPC-induced VSMC contraction in co-culture systems. Therefore, in vitro co-culture systems could be used to study the underlying mechanisms by which VECs inhibit SPC-induced VSMC contraction.

We have previously reported that integrin $\beta 4$ is a very important membrane protein in VEC apoptosis, senescence and differentiation ${ }^{[10-12]}$. The data from the present study suggest that integrin $\beta 4$ is also a key factor in the VEC-mediated 

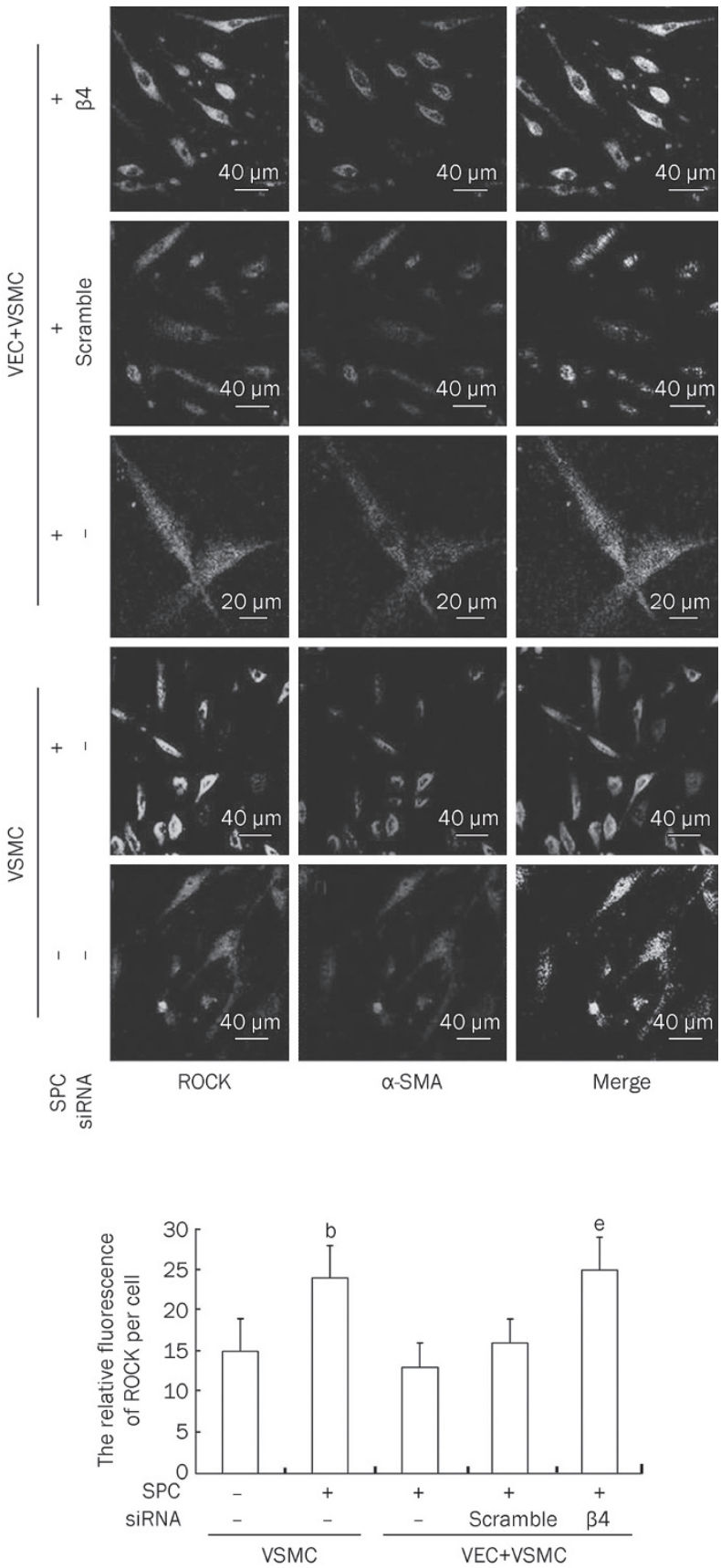

Figure 6. Effects of integrin $\beta 4$ knockdown in VECs on changes in ROCK levels in co-cultured VSMCs. VSMCs that had been co-cultured with VECs that were treated with or without integrin $\beta 4$-specific siRNA ( $\beta 4$ ) or scramble control SiRNA in the presence of SPC were immunostained for ROCK, $\alpha$-SMA, or both. The bar graph shows the relative fluorescence intensity of ROCK per VSMC as determined by laser scanning confocal microscopy $\left({ }^{b} P<0.05\right.$ vs SPC-non-stimulated VSMCs; ${ }^{e} P<0.05$ vs SPCstimulated scramble ctrl in co-cultured system, $n=4$ ).

regulation of SPC-induced VSMC contraction. It is clear that $\mathrm{NO}$ is an important factor for the modulation of VSMC contraction by SPC-stimulated VECs ${ }^{[8]}$. Our current results show that SPC promotes the production of NO in VECs, and the knockdown of integrin $\beta 4$ rescues the levels of NO in VECs that have been stimulated with SPC. Thus, integrin $\beta 4$ may regulate VSMC contraction through the action of NO. We will use NO inhibitors in future studies to verify the role of NO generation in endothelial cells in the reduction of SPC-stimulated VSMC contraction.

It has been reported that Fyn may act as an regulator in coronary artery contraction ${ }^{[25]}$. However, research into the role of Fyn in VECs is limited to endothelial cell migration and capillary morphogenesis ${ }^{[16,26]}$. To date, the possible role of VECderived Fyn in SPC-induced VSMC contraction is not known. In the present study, the siRNA-mediated down-regulation of Fyn in the VECs counteracted the SPC-induced contraction of the VSMCs, suggesting that Fyn is another important factor in the SPC-induced regulation of VSMC contraction by VECs.

The association between integrin $\beta 4$ and Fyn has been analyzed previously. It has been reported that a6 $\beta 4$ integrin selectively activates the Src family member Fyn in response to receptor engagement during tumor progression and promotes this tumor progression ${ }^{[27]}$. However, Wang et al discovered that Fyn phosphorylates the $\beta 4$ cytoplasmic domain and causes the disassembly of hemidesmosomes, which is a prerequisite for normal keratinocyte migration and squamous carcinoma invasion $^{[28]}$. Therefore, the relationship between integrin $\beta 4$ and Fyn remains to be elucidated. Our data show that Fyn levels are regulated by integrin $\beta 4$, but changes in the protein levels that are observed in integrin $\beta 4$-silenced cells in this study may reflect solely the effects of integrin $\beta 4$ silencing on the transcription of the Fyn gene or the degradation of the Fyn protein. The kinase assay and the detection of changes in phosphorylation statuses and post-activation translocation are the generally accepted methods for verifying kinase activation in a signaling cascade. Therefore, the mechanism by which Fyn regulates contraction, in addition to its relationship with integrin $\beta 4$, requires further study.

In the present study, the contraction of the collagen gel when the VSMCs were co-cultured with the scramble-siRNAtransfected VECs amounted to $60 \%-75 \%$ of that of the control (Figure 2D and Figure 3D), and Figure 1C shows that co-culturing with untransfected VECs completely blocks VSMC contraction. Therefore, the effects of the RNAiFect transfection reagent or the inactive siRNA sequence must be acknowledged as was previously described by Kriegel et al ${ }^{[29]}$. However, the use of integrin $\beta 4$ and Fyn siRNA resulted in the statistically significant promotion of collagen gel contraction. Therefore, integrin $\beta 4$ and Fyn in the VECs indeed played important roles in the reduction of VSMC contraction.

In rat aortic smooth muscle cells, the translocation of ROCK from the cytosol to the cell membrane may play a role in SPCinduced contraction ${ }^{[30]}$. In this study, we further observed that the levels of ROCK were associated with the contraction of VSMCs, which may be a marker for indicating VSMC contraction. However, the elucidation of the exact function of ROCK requires further analyses involving kinase activation.

In summary, our results showed that VECs inhibited SPCinduced VSMC contraction in co-culture systems. VECs performed this function using the integrin $\beta 4$ and Fyn proteins. 
NO may be downstream of integrin $\beta 4$ in VECs and assist in performing this function. Furthermore, the levels of ROCK in the VSMCs may be novel markers for the detection of VSMC contraction. The present data provided novel clues for understanding the mechanism by which VECs control the SPCinduced contraction of VSMCs.

\section{Acknowledgements}

This work was supported by the National Natural Science Foundation of China (№ 30570749, 31070999, and 30800398) and National 973 Research Project (№ 2006CB503803). We thank Dr Sei KOBAYASHIC (Yamaguchi University School of Medicine) for his beneficial advice on VSMC contraction research. We also thank Dr Bao-xiang ZHAO (Shandong University) for his critical reading of the manuscript.

\section{Author contribution}

Jun-ying MIAO, Jing ZHAO, and Yun ZHANG designed the research; Di GE, Ning MENG, Le SU, Shang-li ZHANG, and Jing ZHAO performed the research; Shang-li ZHANG analyzed the data; and Jing ZHAO, Di GE, and Ning MENG wrote the paper.

\section{References}

1 Kalsner S. Coronary artery spasm. Multiple causes and multiple roles in heart disease. Biochem Pharmacol 1995; 49: 859-71.

2 Hellstrom HR. Evidence in favor of the vasospastic cause of coronary artery thrombosis. Am Heart J 1979; 97: 449-52.

3 Factor SM, Cho S. Smooth muscle contraction bands in the media of coronary arteries: A postmortem marker of antemortem coronary spasm. J Am Co11 Cardiol 1985; 6: 1329-37.

4 Lin CS, Penha PD, Zak FG, Lin JC. Morphodynamic interpretation of acute coronary thrombosis, with special reference to volcano-like eruption of atheromatous plaque caused by coronary artery spasm. Angiology 1988; 39: 535-47.

5 Hirano K. Current topics in the regulatory mechanism underlying the $\mathrm{Ca}^{2+}$ sensitization of the contractile apparatus in vascular smooth muscle. J Pharmacol Sci 2007; 104: 109-15.

6 Nixon GF, Mathieson FA, Hunter I. The multi-functional role of sphingosylphosphorylcholine. Prog Lipid Res 2008; 47: 62-75.

7 Shirao S, Kashiwagi S, Sato M, Miwa S, Nakao F, Kurokawa T, et al. Sphingosylphosphorylcholine is a novel messenger for Rho-kinasemediated $\mathrm{Ca}^{2+}$ sensitization in the bovine cerebral artery: unimportant role for protein kinase C. Circ Res 2002; 91: 112-9.

8 Mogami K, Mizukami Y, Todoroki-lkeda N, Ohmura M, Yoshida K, Miwa $\mathrm{S}$, et al. Sphingosylphosphorylcholine induces cytosolic $\mathrm{Ca}^{2+}$ elevation in endothelial cells in situ and causes endothelium-dependent relaxation through nitric oxide production in bovine coronary artery. FEBS Lett 1999; 457: 375-80.

9 Tarone G, Hirsch E, Brancaccio M, De Acetis M, Barberis L, Balzac F, et al. Integrin function and regulation in development. Int J Dev Biol 2000; 44: 725-31.

$10 \mathrm{Su}$ L, Zhao J, Zhao BX, Miao JY, Yin DL, Zhang SL. Safrole oxide induced human umbilical vein vascular endothelial cell differentiation into neuron-like cells by depressing the reactive oxygen species level at the low concentration. Biochim Biophys Acta 2006; 1763: 247-53.

11 Miao JY, Araki S, Kaji K, Hayashi H. Integrin beta4 is involved in apoptotic signal transduction in endothelial cells. Biochem Biophys Res Commun 1997; 233: 182-6.

12 Sun C, Liu X, Qi L, Xu J, Zhao J, Zhang Y, et al. Modulation of vascular endothelial cell senescence by integrin $\beta 4$. J Cell Physiol 2010; 225 : 673-81.
13 Thomas SM, Soriano P, Iwamoto A. Specific and redundant roles of Src and Fyn in organizing the cytoskeleton. Nature 1995; 376: $267-$ 71.

14 Cobb BS, Schaller MD, Leu TH, Parsons JT. Stable association of pp60src and pp59fyn with the focal adhesion-associated protein tyrosine kinase, pp125FAK. Mol Cell Biol 1994; 14: 147-55.

15 Heydarkhan-Hagvall S, Helenius G, Johansson BR, Li JY, Mattsson E, Risberg B. Co-culture of endothelial cells and smooth muscle cells affects gene expression of angiogenic factors. J Cell Biochem 2003; 89: 1250-9.

16 Kanda S, Mochizuki Y, Nakamura T, Miyata Y, Matsuyama T, Kanetake $\mathrm{H}$. Pigment epithelium-derived factor inhibits fibroblast-growth-factor2-induced capillary morphogenesis of endothelial cells through Fyn. J Cell Sci 2005; 118: 961-70.

17 Morikage N, Kishi H, Sato M, Guo F, Shirao S, Yano T, et al. Cholesterol primes vascular smooth muscle to induce $\mathrm{Ca}^{2+}$ sensitization mediated by a sphingosylphosphorylcholine-Rho-kinase pathway: possible role for membrane raft. Circ Res 2006; 99: 299-306.

18 Nakao F, Kobayashi S, Mogami K, Mizukami Y, Shirao S, Miwa S, et al. Involvement of Src family protein tyrosine kinases in $\mathrm{Ca}^{2+}$ sensitization of coronary artery contraction mediated by a sphingosylphosphorylcholine-Rho-kinase pathway. Circ Res 2002; 91: 953-60.

19 Leik CE, Willey A, Graham MF, Walsh SW. Isolation and culture of arterial smooth muscle cells from human placenta. Hypertension 2004; 43: 837-40.

20 Jaffe EA, Nachman RL, Becker CG, Minick RC. Culture of human endothelial cells derived from umbilical veins. J Clin Invest 1973; 52: 2745-56.

21 Wang HQ, Bai L, Shen BR, Yan ZQ, Jiang ZL. Coculture with endothelial cells enhances vascular smooth muscle cell adhesion and spreading via activation of beta1-integrin and phosphatidylinositol 3-kinase/Akt. Eur J Cell Biol 2007; 86: 51-62.

22 Bell E, Ivarsson B, Merrill C. Production of a tissue-like structure by contraction of collagen lattices by human fibroblasts of different proliferative potential in vitro. Proc Natl Acad Sci U S A 1979; 76: 1274-8.

23 Fitzgibbon J, Morrison JJ, Smith TJ, O'Brien M. Modulation of human uterine smooth muscle cell collagen contractility by thrombin, Y-27632, TNF alpha and indomethacin. Reprod Biol Endocrinol 2009; 7: 2.

24 Vi L, Feng L, Zhu RD, Wu Y, Satish L, Gan BS, et al. Periostin differentially induces proliferation, contraction and apoptosis of primary Dupuytren's disease and adjacent palmar fascia cells. Exp Cell Res 2009; 315: 3574-86.

25 Nakao F, Kobayashi S, Mogami K, Mizukami Y, Shirao S, Miwa S, et al. Involvement of Src family protein tyrosine kinases in $\mathrm{Ca}^{2+}$ sensitization of coronary artery contraction mediated by a sphingosylphosphorylcholine-Rho-kinase pathway. Circ Res 2002; 91: 953-60.

26 Lamalice L, Houle F, Huot J. Phosphorylation of Tyr 1214 within VEGFR-2 triggers the recruitment of Nck and activation of Fyn leading to SAPK2/p38 activation and endothelial cell migration in response to VEGF. J Biol Chem 2006; 281: 34009-20.

27 Yang X, Dutta U, Shaw LM. SHP2 mediates the localized activation of Fyn downstream of the $\alpha 6 \beta 4$ integrin to promote carcinoma invasion. Mol Cell Biol 2010; 30: 5306-17.

28 Wang H, Leavitt L, Ramaswamy R, Rapraeger AC. Interaction of syndecan and alpha6beta4 integrin cytoplasmic domains: regulation of ErbB2-mediated integrin activation. J Biol Chem 2010; 285: 13569-79.

29 Kriegel C, Amiji M. Oral TNF- $\alpha$ gene silencing using a polymeric microsphere-based delivery system for the treatment of inflammatory bowel disease. J Control Release 2011; 150: 77-86.

30 Davis JR, Giardina JB, Green GM, Alexander BT, Granger JP, Khalil RA. Reduced endothelial NO-cGMP vascular relaxation pathway during TNF - induced hypertension in pregnant rats. Am J Physiol Regul Integr Comp Physiol 2002; 282: R390-9. 